\title{
ВЫБОР ОПРЕДЕЛЯЮЩИХ ПАРАМЕТРОВ ТЕХНИЧЕСКОГО СОСТОЯНИЯ, ОКАЗЫВАЮЩИХ ВЛИЯНИЕ НА ОСТАТОЧНЫЙ СРОК СЛУЖБЫ ТЕПЛООБМЕННОГО ОБОРУДОВАНИЯ
}

\author{
Кулаков Петр Алексеевич1, \\ kulakov.p.a@mail.ru \\ Рубцов Алексей Вячеславович 1 , \\ sunset202@mail.ru
}

Афанасенко Виталий Геннадьевич1, afanasenko.v.g@yandex.ru

\section{Зубкова Ольга Евгеньевна ${ }^{1}$, zubkovaoe@mail.ru}

\author{
Иванова Кристина Кирилловна ${ }^{1}$, \\ kristi99.03.26@mail.ru
}

\author{
Шарипова Регина Ринатовна ${ }^{1}$, \\ regulya0308@mail.ru
}

1 Уфимский государственный нефтяной технический университет, Россия, 450062, г. Уфра, ул. Космонавтов, 1.

\begin{abstract}
Актуальность исследования обусловлена необходимостью определения остаточного ресурса оборудования с достаточной степенью точности и при различных условиях. Развитие математических моделей для оценки механизмов накопления повреждений и фрактического состояния оборудования позволит прогнозировать развитие изменения состояния объекта, учитывая установленные закономерности механизмов повреждения, и определить момент наступления предельного состояния. Цель: предложить метод оценки остаточного ресурса теплообменных аппаратов, учитывающий экспертный интуитивнологический анализ проблемы с количественной оценкой суждений и обработкой результатов.

объекты: теплообменные аппараты, экспертные заключения по теплообменным аппаратам, используемым в нефртепереработке.

Методы: модель информативности Кульбака-Лейблера, показывающая степень информативности отдельных параметров через плотность одной вероятностной меры по отношению к другой, назначение ресурса безопасной эксплуатации при коррозионно-эрозионном износе материала объекта, назначение ресурса безопасной эксплуатации по изменению параметров технического состояния объекта.

Результаты. Поставлена задача оценки остаточного срока службы теплообменного оборудования после проведения технической диагностики на допускаемых технических параметрах в зависимости от суммарного влияния параметров технического состояния оборудования нефтегазопереработки и нефтехимии. Было отобрано 10 технических и технологических показателей теплообменных аппаратов, а оценка выполнялась с использованием такого показателя, как остаточный срок службы теплообменников. Авторами была разработана математическая модель, вычислены диагностические коэфффициенты и информативность показателя для каждого периода, принятого экспертом. Предложен алгоритм оценки остаточного срока службы теплообменных аппаратов на основе меры информативности Кульбака. Показаны оценки остаточного ресурса, выбивающиеся из построенной модели. Сделаны выводы о рациональности использования предлагаемого алгоритма для решения задач данного типа.
\end{abstract}

\section{Ключевые слова:}

Теплообменный аппарат, остаточный срок службы, прогноз, оценка, эксперт, мера Кульбака.

\section{Введение}

Теплообменное оборудование нефтегазоперерабатывающих и нефтехимических производств составляет значительную долю аппаратурного оформления технологических установок и служит для нагрева, охлаждения, испарения, конденсации и т. д. технологических сред. Теплообменные аппараты, используемые в технологических процессах, являются техническими устройствами, применяемыми на опасном производственном объекте, и, следовательно, к ним применяются строгие требования по определению технического состояния в процессе эксплуатации, регла- ментированные нормативными документами в области промышленной безопасности.

После наработки определенного времени теплообменные аппараты проходят процедуру экспертизы промышленной безопасности $[1,2]$, в которую могут входить такие работы, как:

- изучение и анализ технической документации, данных оперативной диагностики;

- неразрушающий и разрушающий контроль [3];

- поверочные расчеты на прочность и устойчивость $[4,5]$;

- прогнозирование остаточного ресурса [6-8];

- испытание на прочность и плотность и т. д. 
В условиях эксплуатации теплообменных аппаратов в коррозионных средах одним из наиболее важнейших этапов по оценке технического состояния является прогнозирование остаточного ресурса теплообменного аппарата. В данном случае доминирующим механизмом повреждения конструктивных элементов теплообменника является коррозионный износ, и расчет остаточного срока службы ведется по изменению толщины стенки элемента.

В основе прогнозирования остаточного ресурса заложен метод, основанный на сравнительном анализе толщин стенок основных конструктивных элементов, а именно: проектной, минимальной измеренной или минимальной вероятной, а также отбраковочной.

Проектная толщина стенки заложена при проектировочном расчете аппарата, минимальную измеренную мы получаем при проведении ультразвуковой толщинометрии, а минимальную вероятную - в результате обработки данных статистическими методами, отбраковочная толщина определяется расчетным методом, а также приведена в нормативных и технических документах. По изменению проектной толщины через определенный интервал наработки аппарата рассчитывается скорость коррозионного износа по минимальной замеренной или минимальной вероятной толщине, а затем рассчитывается остаточный ресурс, подразумевающий, сколько времени должно пройти, чтобы при определенной скорости коррозии минимальная толщина стенки, полученная при помощи измерений, достигла своего отбраковочного значения [9].

Назначаемый срок ресурса зависит от двух определяющих показателей:

1) срок службы аппарата;

2) максимальная скорость коррозии конструктивного элемента.

Остаточный ресурс назначается исходя из минимального расчетного ресурса для конструктивного элемента теплообменного аппарата, но в любом случае продление дальнейшего срока службы не может превышать 10 лет согласно действующим нормативным документам в области промышленной безопасности.

Как правило, методы математики, применяемые в экспертных расчетах, могут позволить оценить верхнюю границу остаточного ресурса. В некоторых случаях эта граница завышена в разы по отношению к реальному значению в заключении экспертизы промышленной безопасности [10].

Объемные массивы полученных данных позволяют провести анализ возможных исходов будущих экспертиз, разработать математическую модель, наиболее полно характеризующую рассматриваемый вид технических устройств [10-16].

Технические параметры дают представление о состоянии теплообменных аппаратов в различных интервалах времени, а также о процессах, влекущих многообразные траектории и формы износа и вероятные сценарии разрушения [16-19].

В настоящее время широко применяется оценка влияния технических параметров теплообменных систем $[11,20]$, остаточный ресурс моделируется при помощи вычислительной техники $[11,21]$, математики $[16,20,22]$, находят применение нейронные сети [14-16]. Однако даже такой обширный арсенал не всегда дает требуемую точность исходов и возможность применимости сложных уравнений.

Было отобрано 10 признаков, влияющих на остаточный ресурс теплообменных систем: дата ввода в эксплуатацию $\left(\tau_{1}\right)$, дата заключения экспертизы $\left(\tau_{2}\right)$, обечайка корпуса (диаметр внутренний) $(D)$, толщина стенки по паспорту $\left(h_{1}\right)$, длина (высота) $(L)$, толщина стенки минимальная $\left(h_{2}\right)$, срок эксплуатации $\left(\tau_{3}\right)$, скорость коррозии в год $(\varepsilon)$, температура $(T)$, давление $(P)$.

Для проведения оценки были выбраны экспертные заключения по 35 теплообменным аппаратам с примерно одинаковыми условиями эксплуатации по параметрам теплоносителя (табл. 1). В выборке присутствуют экспертные заключения от 3 до 10 лет, но отсутствуют данные по теплообменникам, для которых экспертиза давала бы отрицательное заключение.

Влияние перечисленных параметров $\left(\tau_{1}, \tau_{2}, D, h_{1}, L\right.$, $\left.h_{2}, \tau_{3}, \varepsilon, T, P\right)$ на остаточный ресурс теплообменников можно определить путём вычисления их информативности [10].

Оценка информативности производится посредством меры Кульбака [10]. Мера Кульбака-Лейблера позволяет разбить вероятностное многомерное пространство признаков по показателю интеграла от логарифма плотности одной вероятностной меры по отношению к другой. Поэтому будем производить оценку за несколько итераций: на первой итерации разделим теплообменники на две группы - имеющие остаточный ресурс 5 лет и не имеющие такого ресурса, на второй итерации разделим по остаточному ресурсу 6 лет, на третьей -8 лет и на четвертой итерации - 10 лет согласно экспертным заключениям.

Имеется десять признаков $\left(\tau_{1}, \tau_{2}, D, h_{1}, L, h_{2}, \tau_{3}, \varepsilon, T\right.$, $P)$, а также результат - остаточный ресурс для первой итерации 5 лет либо его отсутствие. Разделим теплообменники на две группы: «А» - имеющие остаточный ресурс 5 и более лет; «В»- не имеющие такого ресурса. В качестве данных для построения модели возьмем теплообменники 1-29, а для проверки модели - теплообменники 30-35.

Находим информативности каждого из десяти признаков.

Рассмотрим пример расчета информативности по признаку «Дата ввода в эксплуатацию», который обозначен $\tau_{1}$.

Диапазон изменения данного параметра от 1957 до 2003 гг. Все значения разбиваем на интервалы: [1957; 1960], (1960; 1970], (1970; 1980], (1980; 1990], (1990; 2000], (2000; 2003]. Имея шесть интервалов, определяем частоту попадания теплообменников в одну из групп («А» или «В»). В нашей выборке всего один теплообменник с датой ввода в эксплуатацию между 1957 и 1960 гг., который попадает в группу «А», а в группу «В» из данного периода попадает 0 теплообменников. В интервале (1980; 1990] имеется десять теплообменников из группы «А» и только один теплообменник из группы «В». 
Известия Томского политехнического университета. Инжиниринг георесурсов. 2020. Т. 331. № 1. 97-105

Кулаков П.А. и др. Выбор определяющих параметров технического состояния, оказывающих влияние на остаточный срок ...

Таблица 1. Данные по теплообменникам и показателям, влияющим на остаточный ресурс

Table 1. Data on heat exchangers and factors affecting the residual life

\begin{tabular}{|c|c|c|c|c|c|c|c|c|c|c|c|c|}
\hline \multirow[b]{2}{*}{$\begin{array}{l}\text { № } \\
\text { No. }\end{array}$} & \multicolumn{2}{|c|}{$\begin{array}{c}\text { Дата (год) } \\
\text { Date of (year) } \\
\end{array}$} & \multirow{2}{*}{ 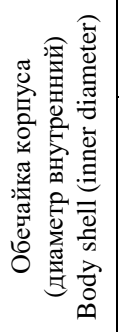 } & \multicolumn{2}{|c|}{$\begin{array}{l}\text { Толщина стенки } \\
\text { Wall thickness }\end{array}$} & \multirow[b]{2}{*}{ 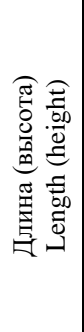 } & \multirow[b]{2}{*}{ 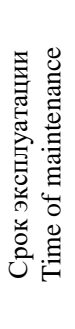 } & \multirow[b]{2}{*}{ 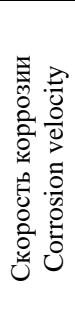 } & \multirow[b]{2}{*}{ 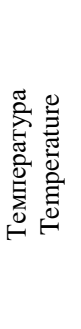 } & \multirow[b]{2}{*}{ 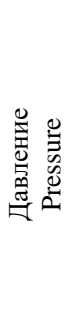 } & \multicolumn{2}{|c|}{$\begin{array}{l}\text { Остаточный ресурс (лет) } \\
\text { Residual resource (years) }\end{array}$} \\
\hline & 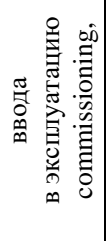 & 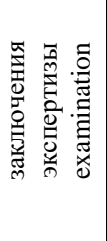 & & 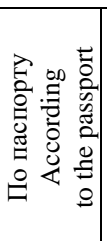 & 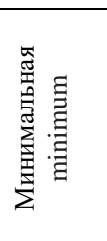 & & & & & & 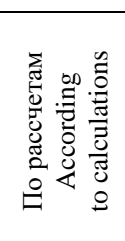 & 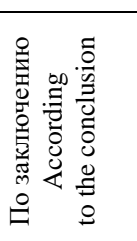 \\
\hline 1 & 1963 & 2004 & 255 & 9 & 6,9 & 6070 & 41 & 0,57 & 150 & 0,6 & 8,2 & 3 \\
\hline 2 & 1987 & 2003 & 1000 & 10 & $\begin{array}{l}7,8 \\
\end{array}$ & 250 & 16 & 1,38 & 150 & 0,4 & 11 & 8 \\
\hline 3 & 1982 & 2004 & 1050 & 8 & 6,3 & 166 & 22 & 0,97 & 200 & 0,75 & 8,2 & 8 \\
\hline 4 & 1989 & 2004 & 600 & 8 & 7,5 & 280 & 15 & 0,42 & 90 & 0,4 & 14,66 & 8 \\
\hline 5 & 1972 & 2003 & 255 & 9 & 5 & 400 & 31 & 1,43 & 126 & 0,25 & 10 & 3 \\
\hline 6 & 1980 & 2008 & 1200 & 10 & 7,4 & 315 & 28 & 0,93 & 200 & 0,6 & 29 & 4 \\
\hline 7 & 1989 & 2005 & 1200 & 10 & 8,1 & 600 & 16 & 1,19 & 60 & 0,6 & 9 & 8 \\
\hline 8 & 1957 & 2006 & 258 & 22 & 16,7 & 129 & 49 & 0,49 & 145 & 8 & 14 & 6 \\
\hline 9 & 1960 & 2008 & 2000 & 10 & 8,1 & 368 & 48 & 0,40 & 340 & 0,6 & 8 & 4 \\
\hline 10 & 1996 & 2010 & 60,3 & 5,54 & 4,2 & 1902 & 14 & 1,73 & 167 & 0,45 & 17 & 4 \\
\hline 11 & 1981 & 2006 & 800 & 10 & 8,8 & 6000 & 25 & 0,48 & 170 & 8 & 6,1 & 6 \\
\hline 12 & 1975 & 2005 & 406 & 10 & 6,9 & 3444 & 30 & 1,03 & 120 & 0,4 & 8 & 8 \\
\hline 13 & 1975 & 2008 & 1200 & 20 & 17,4 & 4000 & 33 & 0,39 & 20 & 2,5 & 10 & 4 \\
\hline 14 & $\begin{array}{ll}1996 \\
\end{array}$ & 2006 & 60,3 & 5,54 & 4,3 & 1902 & 10 & 2,24 & 167 & 0,45 & 9 & 4 \\
\hline 15 & 2000 & 2010 & 1400 & 16 & 15 & 3400 & 10 & 0,63 & 0 & 0,1 & 14,9 & 8 \\
\hline 16 & 1982 & 2003 & 800 & 6 & 5,1 & 485 & 21 & 0,71 & 200 & 0,75 & 10,4 & 10 \\
\hline 17 & 1977 & 2002 & 1000 & 10 & 6,6 & 285 & 25 & 1,36 & 0 & 0,35 & 9,6 & 4 \\
\hline 18 & 1998 & 2005 & 1200 & 8 & 6,4 & 4000 & 7 & 2,86 & 90 & 0,4 & 34 & 8 \\
\hline 19 & 1977 & 2002 & 1000 & 10 & 6,6 & 550 & 25 & 1,36 & 0 & 0,35 & 6 & 4 \\
\hline 20 & 2003 & 2006 & 800 & 10 & 8,8 & 6000 & 3 & 4,00 & 200 & 8 & 8,76 & 6 \\
\hline 21 & 1963 & 2005 & 784 & 8 & 5,6 & 240 & 42 & 0,71 & 100 & 0,6 & 12 & 5 \\
\hline 22 & 1996 & 2006 & 800 & 10 & 8 & 530 & 10 & 2,00 & 100 & 0,4 & 11 & 10 \\
\hline 23 & 1989 & 2006 & 800 & 10 & 7,2 & 400 & 17 & 1,65 & 0 & 8 & 6 & 6 \\
\hline 24 & 1991 & 2010 & 1200 & 12 & 10,5 & 700 & 19 & 0,66 & 200 & 1,8 & 22 & 4 \\
\hline 25 & 1970 & 2008 & 600 & 6 & 5,4 & 1444 & 38 & 0,26 & 0 & 0,4 & 7 & 4 \\
\hline 26 & 1975 & 2008 & 1200 & 20 & 17,4 & 4000 & 33 & 0,39 & 164 & 4 & 9,6 & 4 \\
\hline 27 & 1986 & 2006 & 800 & 16 & 12,2 & 200 & 20 & 1,19 & 220 & 8 & 12 & 6 \\
\hline 28 & 1968 & 2006 & 600 & 22 & 18,8 & 395 & 38 & 0,38 & -18 & 4 & 21 & 6 \\
\hline 29 & 1989 & 2006 & 800 & 16 & 13,2 & 200 & 17 & 1,03 & 0 & 8 & 9 & 6 \\
\hline 30 & 1982 & 2004 & 406 & 4 & 2,8 & 166 & 22 & 1,36 & 147 & 1,6 & 19 & 5 \\
\hline 31 & 1985 & 2005 & 600 & 6 & 5,1 & 180 & 20 & 0,75 & 90 & 0,6 & 18 & 10 \\
\hline 32 & 1982 & 2003 & 406 & 4 & 2,8 & 166 & 21 & 1,43 & 147 & 1,6 & 6 & 5 \\
\hline 33 & 1980 & 2006 & 114 & 6 & 5,8 & 1768 & 26 & 0,14 & 55 & 1,4 & 8 & 4 \\
\hline 34 & 1982 & 2010 & 1200 & 12 & 11 & 350 & 28 & 0,30 & 200 & 1,8 & 40 & 4 \\
\hline 35 & 1978 & 2005 & 800 & 8 & 6,9 & 4195 & 27 & 0,51 & 200 & 8 & 9 & 8 \\
\hline
\end{tabular}

Определяем относительную частоту попадания в ту или иную группу в пределах интервала: если для группы «А» попало 2 теплообменника из 17 теплооб-

менников группы «А», то для первого интервала относительная частота попадания в группу «А» равна $y_{A}=11,77 \%$ (табл. 2).

Таблица 2. Информативность признака "Дата ввода в эксплуатацию»

Table 2. Information content of the feature «Date of commissioning»

\begin{tabular}{|c|c|c|c|c|c|c|c|c|c|c|}
\hline \multirow[t]{2}{*}{$\begin{array}{c}\text { Интервал } \\
\text { Interval }\end{array}$} & \multirow{2}{*}{\begin{tabular}{c|} 
Диапазон \\
изменения \\
T, \% \\
Range \\
of T change, $\%$
\end{tabular}} & \multicolumn{2}{|c|}{$\begin{array}{c}\text { Количество } \\
\text { теплообменников в группе } \\
\text { Number of heat exchangers } \\
\text { in the group } \\
\end{array}$} & \multicolumn{2}{|c|}{$\begin{array}{c}\text { Относительная } \\
\text { частота, \% } \\
\text { Relative frequency, \% }\end{array}$} & \multicolumn{2}{|c|}{$\begin{array}{c}\text { Сглаженная } \\
\text { частота, \% } \\
\text { Smoothed } \\
\text { frequency, \% } \\
\end{array}$} & \multirow[t]{2}{*}{$\frac{\tilde{y}_{A i}}{\tilde{y}_{B i}}$} & \multirow[t]{2}{*}{$\begin{array}{l}\text { ДК } \\
\mathrm{DK}\end{array}$} & \multirow[t]{2}{*}{$J_{i}$} \\
\hline & & $\mathrm{A}$ & $\mathrm{B}$ & $y_{A}$ & $y_{B}$ & $\tilde{y}_{A}$ & $\tilde{y}_{B}$ & & & \\
\hline 1 & До 1960 & 1 & 0 & 5,88 & 0,00 & 5,29 & 8,33 & 0,64 & $-1,97$ & 0,03 \\
\hline 2 & $1960-1970$ & 2 & 2 & 11,77 & 16,67 & 12,35 & 17,50 & 0,71 & $-1,51$ & 0,04 \\
\hline 3 & 1970-1980 & 1 & 6 & 5,88 & 50,00 & 17,06 & 27,50 & 0,62 & $-2,07$ & 0,11 \\
\hline 4 & $1980-1990$ & 9 & 1 & 52,94 & 8,33 & 27,06 & 20,00 & 1,35 & 1,31 & 0,05 \\
\hline 5 & 1990-2000 & 2 & 3 & 11,77 & 25,00 & 18,24 & 16,67 & 1,09 & 0,39 & 0,00 \\
\hline 6 & От 2000 & 2 & 0 & 11,77 & 0,00 & 12,35 & 5,83 & 2,12 & 3,26 & 0,11 \\
\hline \multicolumn{2}{|c|}{ Сумма/Amount } & 17 & 12 & 100 & 100 & - & - & $\begin{array}{llll}- & & & \end{array}$ & - & 0,33 \\
\hline
\end{tabular}


Определение взвешенной сглаженной частоты имеет целью нивелирование влияния распределения на интервалы. Необходимо учитывать частоту признака в двух интервалах предшествующих и двух интервалах последующих. Интервалы, которые предшествуют интервалу № 1, - нулевой и минус первый - имеют нулевую частоту.

Для компенсации субъективного влияния распределения на интервалы находится коэффициент - взвешенная сглаженная частота. В нем учитываются по два предшествующих и последующих интервала с эмпирическими коэффициентами, зависящими от числа интервалов и от количества теплообменников в выборке. При расчете интервала № 1 предыдущие интервалы, а также последующие интервалы для последнего интервала имеют частоту, равную нулю.

Взвешенную сглаженную частоту находим по формуле [10]:

$$
\tilde{y}=\frac{\left(y_{1}+2 \cdot y_{2}+4 \cdot y_{3}+2 \cdot y_{4}+y_{5}\right)}{10},
$$

где $y_{1} \ldots y_{5}$ - частоты в интервалах.

Значение взвешенной сглаженной частоты в первом интервале для группы «А» равно:

$$
\tilde{y}_{\mathrm{A} 1}=\frac{(0+2 \cdot 0+4 \cdot 5,88+2 \cdot 11,77+5,88)}{10}=5,29 \% \text {. }
$$

Значение взвешенной сглаженной частоты в первом интервале для группы «В» равно:

$$
\tilde{y}_{\mathrm{B} 1}=\frac{(0+2 \cdot 0+4 \cdot 0+2 \cdot 16,67+50)}{10}=8,33 \% .
$$

Значение взвешенной сглаженной частоты в третьем интервале для группы «В» равно:

$$
\tilde{y}_{\mathrm{B} 3}=\frac{(0+2 \cdot 16,67+4 \cdot 50+2 \cdot 8,33+25)}{10}=27,5 \% .
$$

Для каждого из интервалов определяем значение соотношения сглаженных частот. Для первого интервала:

$$
\frac{\tilde{y}_{\mathrm{A} 1}}{\tilde{y}_{\mathrm{B} 1}}=\frac{5,29}{8,33}=0,64 \text {. }
$$

Для каждого интервала находим значение диагностического коэффициента (ДК) [17]:

$$
\text { ДК }{ }_{i}=10 \cdot \lg \frac{\tilde{y}_{\mathrm{A} i}}{\tilde{y}_{\mathrm{B} i}} .
$$

Значение динамического коэффициента на первом интервале:

$$
\text { ДК }{ }_{1}=10 \cdot \lg 0,64=-1,97 .
$$

По формуле Кульбака коэффициент информативности признака в $i$-м интервале [17]:

$$
J_{i}=0,5 \cdot Д \kappa_{i} \cdot\left(\tilde{y}_{\mathrm{A} i}-\tilde{y}_{\mathrm{B} i}\right) / 100 .
$$

Значение коэффициента информативности $J_{i}$ для первого интервала:

$$
J_{1}=0,5 \cdot(-1,97) \cdot(5,29-8,33) / 100=0,03 .
$$

Информативность признака 1 определяется суммой коэффициентов информативности по всем интервалам. Результаты определения информативности всех десяти признаков приведены в табл. 3 .

Наибольшей информативностью $(J=1,287)$ обладает признак «Обечайка корпуса», а наименьшей информативностью $(J 0,9)$-признак «Толщина стенки по минимальная».

По каждому теплообменнику суммировали диагностические коэффициенты [23]. Распределения сумм диагностических признаков для теплообменников, которые обладают и не обладают остаточным ресурсом в 5 лет, показаны на рис. 1 .

По аналогии с исследованными выше теплообменниками и используя полученные диагностические коэффициенты, произведем оценку остаточного ресурса семи теплообменников из контрольного набора.

Из рис. 1 видно, что при сумме диагностических коэффициентов менее -6 , остаточный ресурс менее 5 лет, при сумме диагностических коэффициентов более -2 , остаточный ресурс более 5 лет, на промежутке $[-6,-3]$ требуются дополнительные расчеты.

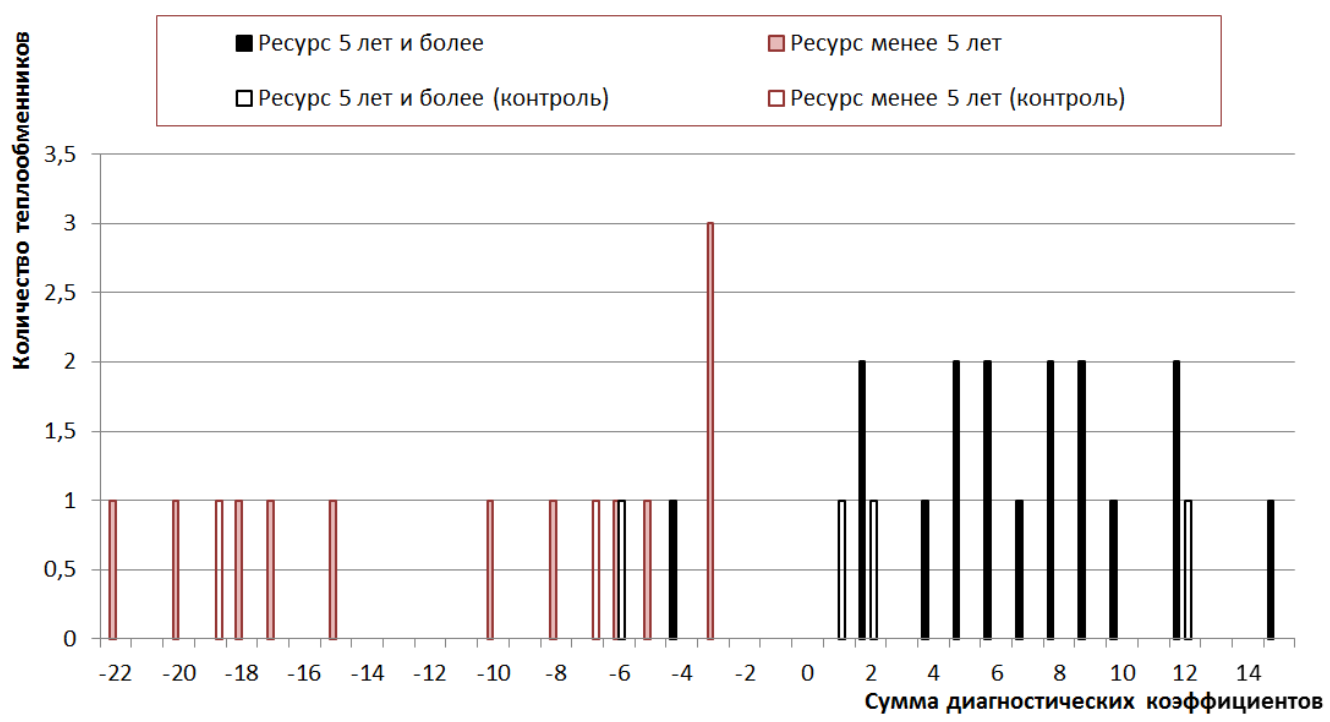

Рис. 1. Распределение теплообменников по сумме диагностических коэффициентов для остаточного ресурса 5 лет Fig. 1. Distribution of heat exchangers by the sum of diagnostic factors for a residual life of 5 years 
Известия Томского политехнического университета. Инжиниринг георесурсов. 2020. Т. 331. № 1. 97-105

Кулаков П.А. и др. Выбор определяющих параметров технического состояния, оказывающих влияние на остаточный срок ...

Таблица 3. Результаты определения диагностических коэффициентов и информативности признаков

Table 3. Results of determining the diagnostic coefficient and informative signs

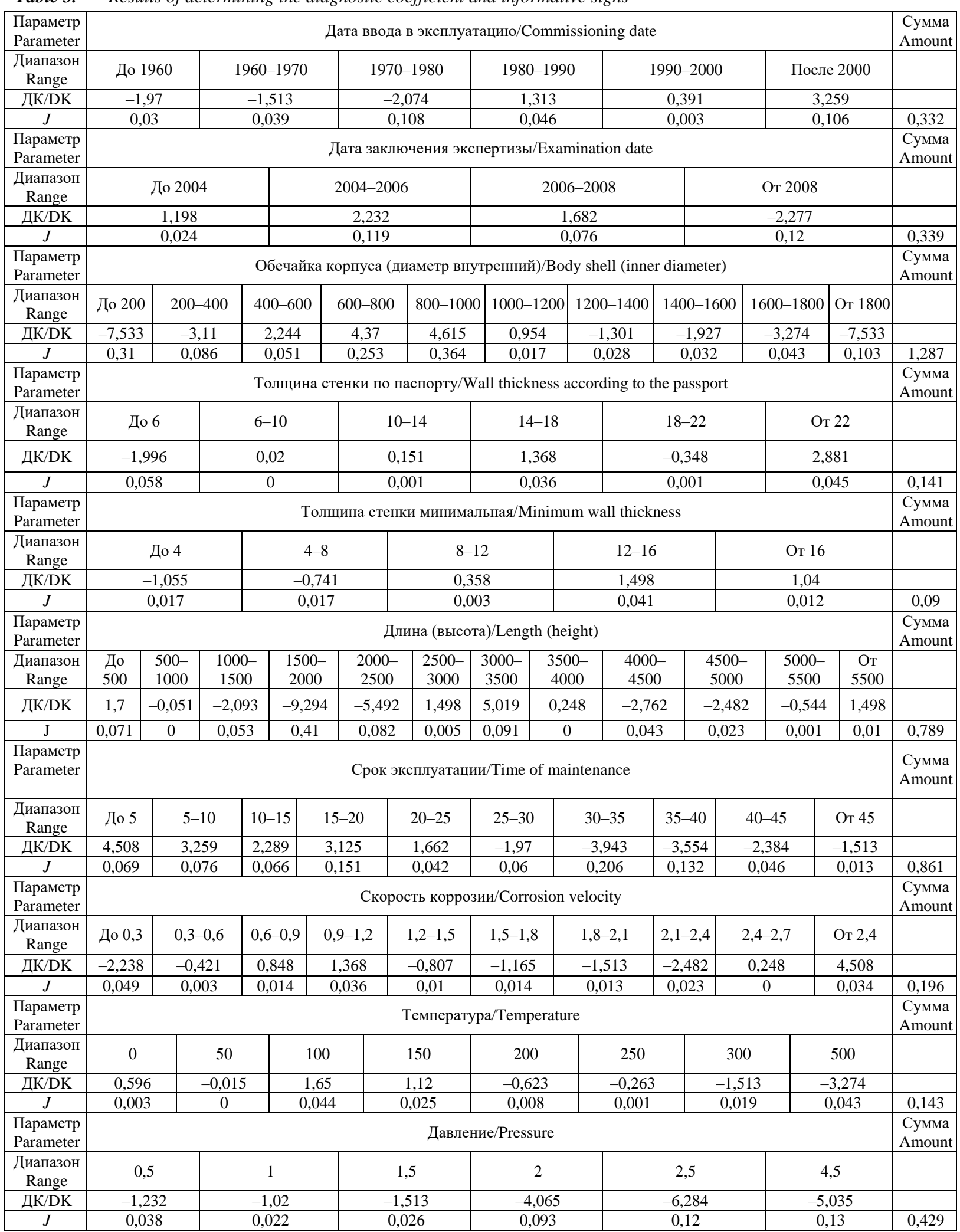

Далее применили данный алгоритм для построения модели и оценки возможного остаточного ресурса теплообменников, равного 6, 8 и 10 лет.

Сопоставив результаты моделирования с экспертным заключением, выявили, что для горизонта пла- нирования 5 лет выявлено 4 теплообменных аппарата (№ $6,8,19,24)$ из 35, которые попадают в зону неопределенности и требуют более детального анализа. Теплообменник № 35 выбивается из модели, и расчеты показывают ошибочный результат. 35-й теплооб- 
менный аппарат по техническим характеристикам, согласно модели, может иметь остаточный ресурс до 5 лет вместо 8 лет. Теплообменник 26 показал существенные отклонения в модели при оценке ресурса в 8 лет, в результате чего для теплообменников 22 и 28 поставили статус «Не определено».

Большее количество теплообменников (100 и более) в модели должно повысить точность прогноза, давая возможность более детального анализа интервала неопределенности. С другой стороны, большое влияние оказывают экспертные оценки состояния, нормативная документация, не преобразуемая к числовым показателям, а также предыдущие экспертные заключения. Кроме того, некоторые параметры, такие как, например, рабочая среда в теплообменном аппарате сложно поддается переводу в числовые значения. Предлагаемый подход позволяет учесть нечисловые показатели через анализ экспертных оценок предшествующих заключений.

\section{Заключение}

Достоверность прогнозирования остаточного ресурса теплообменного оборудования зависит от многих технических параметров, которые эксперт определяет на всех этапах проведения экспертизы промышленной безопасности, начиная от изучения и

\section{СПИСОК ЛИТЕРАТУРЬ}

1. Fran R.J., Carsten R., Antonio M. A security engineering proces for systems of systems using security patterns // IEEE Syst Council. Annual IEEE Systems Conference. - Ontario, 2014. - P. 8-11.

2. Molyneux J. Industrial perspective on the seminar: the viewpoint of a mining expert // Springer briefs in Applied Sciences and Technology. - 2018. - P. 97-101.

3. Argandona G., Biezma M.V., Berrueta J.M. Detection of secondary phases in UNS S32760 superduplex stainless steel by destructive and non-destructive techniques // Journal of Materials Engineering and Performance. - 2016. - V. 25. - № 12. - P. 5269-5279.

4. Xu Reng-chong, He Yan-lin, Jiang Hu. Microstructures and mechanical properties of ferrite-based lightweight steel with different compositions // Journal of Iron and Steel Research International. 2017. - V. 24. - № 7. - P. 737-742.

5. Peng Ji-bo, Jiang Hu, Zhang Gong-ting. In-situ analysis of retained austenite transformation in high-performance micro-alloyed TRIP steel // Journal of Iron and Steel Research International. - 2017. V. 24. - № 3. - P. 313-320.

6. Pan Zhipeng, Liang Steven Y., Garmestani Hamid. Finite element simulation of residual stress in machining of Ti-6Al-4V with a microstructural consideration // Proc. of the Institution of Mechanical Engineers. P. B. Journal of Engineering Manufacture. - 2019. V. 233. - № 4. - P. 1103-1111.

7. Moraes J.F.C., Jordon J.B., Su X. Effect of process deformation history on mechanical performance of AM60B to AA6082 selfpierce riveted joints // Engineering Fracture Mechanics. - 2019. V. 209. - P. 92-104.

8. Roxas Cheryl Lyne C., Lejano Bernardo A. An artificial neural network model for the corrosion current density of steel in mortar mixed with seawater // International Journal of Geomate. - 2019. V. 16. - № 56. - P. 79-84

9. Mukhametzyanov Z.R., Rubtsov A.V., Valiev A.S. Estimation of influence character of installation loads on degree of corrosive wear of column apparatus // Proc. of the $4^{\text {th }}$ International Conference on Industrial Engineering. ICIE 2018. Lecture Notes in Mechanical Engineering. - Cham: Springer, 2019. - P. 1999-2006.

10. Кульбак С. Теория информации и статистика. - М.: Наука 1967. $-408 \mathrm{c}$. анализа технической и эксплуатационной документации и заканчивая расчетом ресурса и проведением испытаний. Не всегда полученная информация является достаточно реалистичной и безошибочной для продления срока безопасной эксплуатации с определенной долей вероятности. При оценке остаточного ресурса по стандартным методикам в 22 случаях из 35 результат расчетов превышает в 1,5 раза назначенный, в 15 случаях превышает в 2 раза, а в 3 случаях - в 5 и более раз. Разработанный подход к оценке остаточного срока службы теплообменных аппаратов, приведенный в данной статье, рекомендовано применять в случаях, когда присутствует большая погрешность исходной информации, которая необходима для осуществления экспертной оценки. Построенная математическая модель существенно повышает точность оценки - из контрольной выборки только 2 объекта определены с ошибкой в 2 года, а остальные совпали с оценкой эксперта. Данный способ можно применить и для других видов технологического оборудования трубопроводные системы, резервуары, колонны и т. д. Проанализировав диагностические коэффициенты, имеется возможность установить диапазоны значений исходных параметров, существенно снижающих остаточный ресурс оборудования.

11. Аношкин А.Н., Поспелов А.Б., Якушев Р.М. Особенности деформирования и разрушения комбинированных полимерных труб при низких температурах // Вестник пермского национального исследовательского политехнического университета. Механика. - 2014. - № 2. - С. 5-28.

12. Стеклов А.С., Серебряков А.В., Титов В.Г. Прогнозирование технического состояния судовых электроэнергетических систем // Вестник Ивановского государственного энергетического университета. - 2016. - № 5. - С. 21-26.

13. Котельников В.В., Рыков А.Н., Козельская С.О. Использование нейронных сетей с глубинным обучением для прогнозирования и оценки уровня критичности дефектов конструкций // Промышленные АСУ и контроллеры. - 2016. - № 12. - С. 39-45.

14. Benzaoui Abderrahmane, Kocaefe Duygu, Bhattacharyay Dipankar. A non-destructive technique for the on-line quality control of green and baked anodes // Metals. -2017 . - V. 7. - № 4. P. 128-137.

15. Шкель Ю.С., Щипачев А.М. Определение остаточного ресурса металла с использованием нейронных сетей // Нефтегазопереработка-2015: Материалы Международной научнопрактической конференции. - Уфа, 2015. - Уфа: ГУП «Институт нефтехимпереработки РБ», 2015. - С. 260-262.

16. Kulakov P.A., Apparov I.H.Y., Afanasenko V.G. Improvement of mud pump valve // 2018 IOP Conference Series: Materials Science and Engineering. - 2018. - V. 451. - № 1. - 012201.

17. Богданович А.В. Вероятностная оценка эффективности гравитационного обогащения и математическое планирование экстремальных экспериментов // Обогащение руд. - 2017. № 4 (370). - С. 22-27.

18. Шабельников С.И. Прогноз мест проявления малоамплитудных тектонических разрывов угольных пластов при проходке горных выработок // Горный журнал. - 2017. - № 12. - С. 21-24.

19. Кучерявый В.И., Мильков С.Н. Вероятностная оценка остаточного ресурса газонефтепровода кольцевых напряжений // Проблемы машиностроения и надежности машин. - 2016. № 1. - C. 105-110.

20. Кутлубулатов А.А., Кулаков П.А. Прогнозирование эффективности операций по гидроразрыву пласта // Известия Тульского государственного университета. Науки о Земле. 2017. - № 2. - C. 88-102. 
Известия Томского политехнического университета. Инжиниринг георесурсов. 2020. Т. 331. № 1. 97-105

Кулаков П.А. и др. Выбор определяющих параметров технического состояния, оказывающих влияние на остаточный срок ...

21. Окладникова Е.Н., Сугак Е.В. Оценка остаточного ресурса безопасной эксплуатации с учетом случайных факторов // Сибирский журнал науки и технологий. - 2011. - № 2 (35). C. $132-136$.

22. Kulakov P.A., Kutlubulatov A.A., Afanasenko V.G. Forecasting of the hydraulic fracturing efficiency as components of its design optimization // Socar Proceedings Issue. - 2018. - V. 2. - P. 41-48

\section{Информация об авторах}

Кулаков П.А., кандидат технических наук, доцент кафедры технологических машин и оборудования, руководитель отделения сервисного обслуживания объектов нефтегазовой отрасли Уфимского государственного нефтяного технического университета.

Рубцов A.B., кандидат технических наук, доцент кафедры технологических машин и оборудования Уфимского государственного нефтяного технического университета.

Афанасенко В.Г., кандидат технических наук, доцент кафедры технологических машин и оборудования Уфимского государственного нефтяного технического университета.

Зубкова O.E., кандидат технических наук, доцент кафедры механики конструирования машин Уфимского государственного нефтяного технического университета.

Иванова К.К., студент Уфимского государственного нефтяного технического университета.

Шарипова P.P., кандидат технических наук, доцент кафедры механики конструирования машин Уфимского государственного нефтяного технического университета. 
UDC 681

\title{
SELECTION OF DETERMINING PARAMETERS OF TECHNICAL CONDITION AFFECTING THE RESIDUAL LIFE OF THE HEAT EXCHANGING EQUIPMENT
}

Petr A. Kulakov',

kulakov.p.a@mail.ru

\author{
Aleksey V. Rubtsov ${ }^{1}$, \\ sunset202@mail.ru
}

Vitalii G. Afanasenko1, afanasenko.v.g@yandex.ru

Olga E. Zubkova ${ }^{1}$ zubkovaoe@mail.ru

\author{
Kristina K. Ivanova1, \\ kristi99.03.26@mail.ru \\ Regina R. Sharipova ${ }^{1}$, \\ regulya0308@mail.ru \\ 1 Ufa State Petroleum Technological University, \\ 1, Kosmonavtov street, Ufa, 450062, Russia.
}

The relevance of the research is caused by the necessity to determine the residual resource of the equipment with a sufficient degree of accuracy and in various circumstances. The development of mathematical models for assessing the mechanisms of damage accumulation and the actual state of the equipment will allow predicting the development of changes in the state of the object, considering the established patterns of damage mechanisms, and determining the moment of occurrence of the limit state.

The aim of the research is to propose a method for assessing the residual resource of heat exchangers, considering expert intuitive-logical analysis of the problem with a quantitative assessment of judgments and processing of results.

Objects: heat exchangers, expert conclusions on heat exchangers used in oil refining.

Methods: Kullback-Leibler informative model, showing the degree of informativeness of individual parameters to the degree of discrepancy between the two classes of objects, diagnosing residual resource for the development of corrosion damage, diagnosing residual resource of equipment related to changed parameters of its technical condition.

Results. The paper sets the task to assess the service life of the heat exchanging equipment after diagnosing on the allowed technical parameters depending on the total effect of the parameters of the technical condition of the equipment for oil and gas processing and petrochemistry. 10 technical and technological indicators of heat exchangers were selected, and the evaluation was performed by using such an indicator as the residual service life of heat exchangers. The authors developed a mathematical model, calculated diagnostic coefficients and informativeness of the indicator for each period accepted by the expert. An algorithm for estimating the residual service life of heat exchangers based on the Kullback informativeness measure is proposed. Estimates of the residual resource, out of the constructed model are shown. Conclusions about the rationality of using the proposed algorithm for solving problems of this type are made.

\section{Key words:}

Heat exchanger, residual life, forecast, evaluation, expert, Kullback measure.

\section{REFERENCES}

1. Ruiz Jose Fran, Rudolph Carsten, Mana Antonio. A security engineering process for systems of systems using security patterns. IEEE Syst Council. Annual IEEE Systems Conference. Ontario, 2014. pp. 8-11.

2. Molyneux J. Industrial perspective on the seminar: the viewpoint of a mining expert. Springer briefs in Applied Sciences and Technology, 2018, pp. 97-101.

3. Argandona G., Biezma M.V., Berrueta J.M. Detection of secondary phases in UNS S32760 superduplex stainless steel by destructive and non-destructive techniques. Journal of Materials Engineering and Performance, 2016, vol. 25, no. 12, pp. 5269-5279.

4. Xu Reng-chong, He Yan-lin; Jiang Hu. Microstructures and mechanical properties of ferrite-based lightweight steel with different compositions. Journal of Iron and Steel Research International, 2017, vol. 24, no. 7, P. 737-742.

5. Peng Ji-bo, Jiang Hu, Zhang Gong-ting. In-situ analysis of retained austenite transformation in high-performance microalloyed TRIP steel. Journal of Iron and Steel Research International, 2017, vol. 24, no. 3, pp. 313-320.
6. Pan Zhipeng, Liang Steven Y., Garmestani Hamid. Finite element simulation of residual stress in machining of Ti-6Al-4V with a microstructural consideration. Proc. of the Institution of Mechanical Engineers. P. B. Journal of Engineering Manufacture, 2019, vol. 233, no. 4, pp. 1103-1111.

7. Moraes J.F.C., Jordon J.B., Su X. Effect of process deformation history on mechanical performance of AM60B to AA6082 selfpierce riveted joints. Engineering Fracture Mechanics, 2019, vol. 209, pp. 92-104

8. Roxas Cheryl L.C., Lejano B.A. An artificial neural network model for the corrosion current density of steel in mortar mixed with seawater. International Journal of Geomate, 2019, vol. 16, no. 56 , pp. 79-84.

9. Mukhametzyanov Z.R., Rubtsov A.V., Valiev A.S. Estimation of influence character of installation loads on degree of corrosive wear of column apparatus. Proc. of the $4^{\text {th }}$ International Conference on Industrial Engineering. ICIE 2018. Lecture Notes in Mechanical Engineering. Cham, Springer, 2019. pp. 1999-2006.

10. Kulbak S. Teoria informatsii $i$ statistiki [Theory of information and statistics]. Moscow, Nauka Publ., 1967. 408 p.

11. Anoshkin A.N., Pospelov A.B., Yakushev R.M. Features of deformation and destruction of combined polymer pipes at low 
temperatures. Bulletin of the Perm National Research Polytechnic University. Mechanics, 2014, no. 2, pp. 5-28. In Rus.

12. Steklov A.S., Serebrakov A.V., Titov V.G. Forecasting the technical condition of the ship electric power systems. Bulletin of Ivanovo State Energy University, 2016, no. 5, pp. 21-26. In Rus.

13. Kotelnikov V.V., Rikov A.N., Kozelskaya S.O. The usage of neural networks with deep learning to predict and evaluate the critical level of structural defects. Industrial control systems and controllers, 2016, no. 12, pp. 39-45. In Rus.

14. Benzaoui Abderrahmane, Kocaefe Duygu, Bhattacharyay Dipankar. A non-destructive technique for the on-line quality control of green and baked anodes. Metals, 2017, vol. 7, no. 4 pp. 128-137.

15. Shkel Yu.S., Shchipachev A.M. Opredelenie ostatochnogo resursa metalla s ispolzovaniem neyronnykh setey [Determination of the residual metal resource using neural networks]. Neftegazopererabotka-2015. Materialy Mezhdunarodnoy nauchnoprakticheskoy konferentsii [Oil and Gas Processing-2015. Materials of the International Scientific and Practical Conference]. Ufa 2015. Ufa, Institute of Petrochemical Processing RB, 2015. pp. 260-262.

16. Kulakov P.A., Apparov I.H.Y., Afanasenko V.G. Improvement of mud pump valve. IOP Conference Series: Materials Science and Engineering, 2018, vol. 451, no. 1, no. 012201.
17. Bogdanovich A.V. Probabilistic estimation of the efficiency of gravity enrichment and mathematical planning of extreme experiments. Ore dressing, 2017, no. 4, pp. 22-27. In Rus.

18. Shabelnikov S.I. Prediction of low-amplitude tectonic ruptures of coal seams during mining. Mining journal, 2017, no. 12, pp. 21-24. In Rus.

19. Kucheraviy V.I., Milkov S.N. Probabilistic assessment of the residual life of the ring and gas pipeline oil and gas pipeline. Problems of mechanical engineering and machine reliability, 2016, no. 1, pp. 105-110. In Rus.

20. Kutlubulatov A. A., Kulakov P. A. Forecasting the efficiency of operations for hydraulic fracturing. Proceedings of the Tula state university. Science of earth, 2017, vol. 2, pp. 88-102.

21. Okladnikova E. N., Sugak E.V. Assessment of the residual resource of safe operation considering random factors. Siberian Journal of Science and Technology, 2011, no. 2, pp. 132-136. In Rus.

22. Kulakov P.A., Kutlubulatov A.A., Afanasenko V.G. Forecasting of the Hydraulic Fracturing Efficiency as Components of Its Design Optimization. Socar Proceedings Issue, 2018, vol. 2, pp. 41-48. In Rus.

23. Bolstad W.M. Introduction to Bayesian Statistics. New Jersey, Wiley, 2007. $463 \mathrm{p}$.

Received: 2 December 2019.

\section{Information about the authors}

Petr A. Kulakov, Cand. Sc., associate professor, Ufa State Petroleum Technological University.

Aleksey V. Rubtsov, Cand. Sc., asociate professor, Ufa State Petroleum Technological University.

Vitalii G. Afanasenko, Cand. Sc., associate professor, Ufa State Petroleum Technological University.

Olga E. Zubkova, Cand. Sc., associate professor, Ufa State Petroleum Technological University.

Kristina K. Ivanova, student, Ufa State Petroleum Technological University.

Regina R. Sharipova, Cand. Sc., associate professor, Ufa State Petroleum Technological University. 